July 19, 2000

BI-TP 2000/26

\title{
Phase Transitions in QCD
}

\author{
Helmut Satz \\ Fakultät für Physik, Universität Bielefeld \\ D-33501 Bielefeld, Germany
}

\begin{abstract}
At high temperatures or densities, hadronic matter shows different forms of critical behaviour: colour deconfinement, chiral symmetry restoration, and diquark condensation. I first discuss the conceptual basis of these phenomena and then consider the description of colour deconfinement in terms of symmetry breaking, through colour screening and as percolation transition.
\end{abstract}

\section{States of Matter}

Hadronic matter is endowed with an inherent density limit. The usual hadrons have an intrinsic size $r_{h} \simeq 1 \mathrm{fm}$, so that a hadron needs a space of volume $V_{h} \simeq(4 \pi / 3) r_{h}^{3}$ to exist. Therefore

$$
n_{c} \simeq\left(1 / V_{h}\right) \simeq 0.25 \mathrm{fm}^{-3} \simeq 1.5 n_{0}
$$

is the limiting density for such a medium; here $n_{0}=0.17 \mathrm{fm}^{-3}$ denotes the density of normal nuclear matter. In turn, this also leads to a limiting temperature for hadronic matter,

$$
T_{c} \sim\left(1 / r_{h}\right) \sim 0.2 \mathrm{GeV},
$$

as pointed out by Pomeranchuk almost fifty years ago [1]. Considering an ideal gas of resonances, whose composition was based on a classical partitioning problem [2], Hagedorn found a very similar limit, which he proposed as the ultimate temperature of strongly interacting matter [3]. Dual resonance dynamics led to an essentially equivalent composition law 四. Soon afterwards Cabibbo and Parisi noted that the 'limit' more likely corresponded to a critical point, signalling the transition to a new state of matter, the quark-gluon plasma [5]. What remains is the realization that, on geometric, combinatoric or dynamic grounds, hadron thermodynamics defines its own limit.

\footnotetext{
${ }^{1}$ Invited talk at the 3rd Catania Relativistic Ion Studies CRIS 2000, Acicastello/Italy, May 22 - 26, 2000; at the XL Cracow School of Theoretical Physics, Zakopane/Poland, June 3 -11, 2000; and at the Conference on Strong and Electroweak Matter SEWM 2000, Marseille/France, June 13 - 17, 2000.
} 
This limit can be approached in two ways. The obvious is to compress cold nuclear matter beyond $n_{0}$; but in relativistic thermodynamics, 'heating' mesonic matter leads to particle production and thus also increases the density. As a result, strongly interacting matter has a $T-\mu_{B}$ phase diagram, where $T$ denotes the temperature and $\mu_{B}$ the chemical potential specifying the overall baryon number density. In the $T-\mu_{B}$ plane, there must thus be a limiting curve for hadronic matter, beyond which the density is too great to allow the existence of hadrons.

Since QCD defines hadrons as bound states of quarks, the general phase structure of strongly interacting matter is quite evident: for densities below $n_{c}$, it consists of colourless hadrons, i.e., colour singlet bound states of three quarks or of a quark-antiquark pair. Above $n_{c}$, deconfinement leads to a medium consisting of coloured constituents. There are three possible forms for such constituents:

- coloured massive quark-gluon states: constituent quarks;

- coloured massive quark-quark states: diquarks;

- coloured massless quarks and gluons: a quark-gluon plasma.

What actually happens in the different regions of the $T-\mu_{B}$ diagram?

Before addressing this question, we have to consider how deconfinement can take place. The confining potential between a static quark and antiquark separated by a distance $r$ has the idealized form

$$
V(r) \sim \sigma r,
$$

where $\sigma \simeq 0.8 \mathrm{GeV} / \mathrm{fm}$ is the string tension. The quarks inside a hadron are therefore confined: the hadron cannot be broken up into its coloured constituents, since this would require an infinite amount of energy.

In a dense medium, however, there is another way to dissociate bound states. The presence of many other charges leads to charge screening, which reduces the range of the forces between charges. A well-known example is Debye screening, which suppresses the long-range part of the Coulomb potential between electric charges,

$$
V(r)=\frac{1}{r} \rightarrow \frac{1}{r} e^{-\mu r},
$$

where $r_{D}=\mu^{-1}$ is the Debye radius, defining the range of the force remaining effective between charges in the medium. When it becomes smaller than the atomic binding radius, an insulator consisting of charge-neutral atoms turns into a conducting plasma of unbound electric charges [6]. In QCD, the corresponding effect leads to colour screening,

$$
V(r)=\sigma r \rightarrow \sigma r\left[\frac{1-e^{-\mu r}}{\mu r}\right],
$$

where $\mu^{-1}$ now defines the colour screening radius?. Deconfinement thus is the insulatorconductor transition of QCD, with colourless bound states as constituents below and

\footnotetext{
${ }^{2}$ The difference in the form of the screening functions in Eq. (州) and (5) is due to the different forms of the unscreened potentials [7].
} 
coloured constituents above the deconfinement point. But what is the nature of the conducting phase here?

One way to study that is to consider the effective quark mass. The input quark masses in the QCD Lagrangian are (for $u$ and $d$ quarks) almost zero, $m_{q} \simeq 0$. In the confined phase, hadrons behave as if they consist of constituent quarks of mass $m_{Q}$, with $m_{n} \simeq 3 m_{Q}$ and $m_{\rho} \simeq 2 m_{Q}$, for nucleons and (non-Goldstone) mesons, respectively. Hence here the quarks manage to 'dress' themselves with gluons to acquire a mass $m_{Q} \simeq 0.3 \mathrm{GeV}$.

At sufficiently high temperatures, thermal motion will presumably 'shake off' the dressing, so that somewhere in the course of the hadron-quark matter transition there will be an effective quark mass shift $m_{Q} \rightarrow m_{q}$. For vanishing $m_{q}$, the QCD Lagrangian is chirally symmetric; hence this chiral symmetry must be spontaneously broken in the confined phase and restored in the hot QGP. One therefore often refers to the shift in effective quark mass $m_{Q} \rightarrow 0$ as chiral symmetry restoration. Such a shift is more general, however, and can occur as well for $m_{q} \neq 0$, as shown by the shift of the effective electron mass between insulator and conductor.

That leads to the next question: is there also a colour superconductor? At low temperatures, collective effects of an electrically conducting medium can overcome the Coulomb repulsion between like charges and lead to a binding of electrons into doubly charged Cooper pairs. These, being bosons, can condensate to form a superconductor. In QCD, the conditions for creating a superconductor are in fact much more favorable. An attractive local potential couples two triplet quark states to a bosonic anti-triplet diquark state, so that in QCD there is a dynamical basis for colour superconductivity through diquark condensation [8].

In very recent years, the low temperature, high baryon density part of the QCD phase diagram has received much renewed attention, resulting in the prediction of different superconducting phases and several transitions [9]. Although of great theoretical interest, this region is for the time being accessible neither to lattice studies nor to experiment. I shall therefore concentrate here on the high temperature, low baryon density region and refer to [9] for a discussion of colour superconductivity.

Taking into account what was said, a first guess of the QCD phase diagram leads to a four-phase structure of the generic form shown Fig. 1; as noted, the 'diquark phase' shown there may well consist of several different superconducting phases. In any case, however, lattice QCD tells us that Fig. 1 is wrong: at $\mu=0$, deconfinement and chiral symmetry restoration coincide, so that there is no constituent quark phase. One of the main points I want to address here is why this is so.

A second guess is shown in Fig. 2, with hadronic, diquark and QGP phases. As far as we know, this one may well be correct; for $\mu_{B}=0$, it is, as we shall see from the results provided by finite temperature lattice QCD.

In the classical study of critical behaviour, the analyticity of the partition function $Z(T, \mu, V)$ in the thermodynamic limit of infinite volume, $V \rightarrow \infty$, governs the phase structure, with critical points defined through the divergence of derivatives of $Z(T, \mu, V)$. 


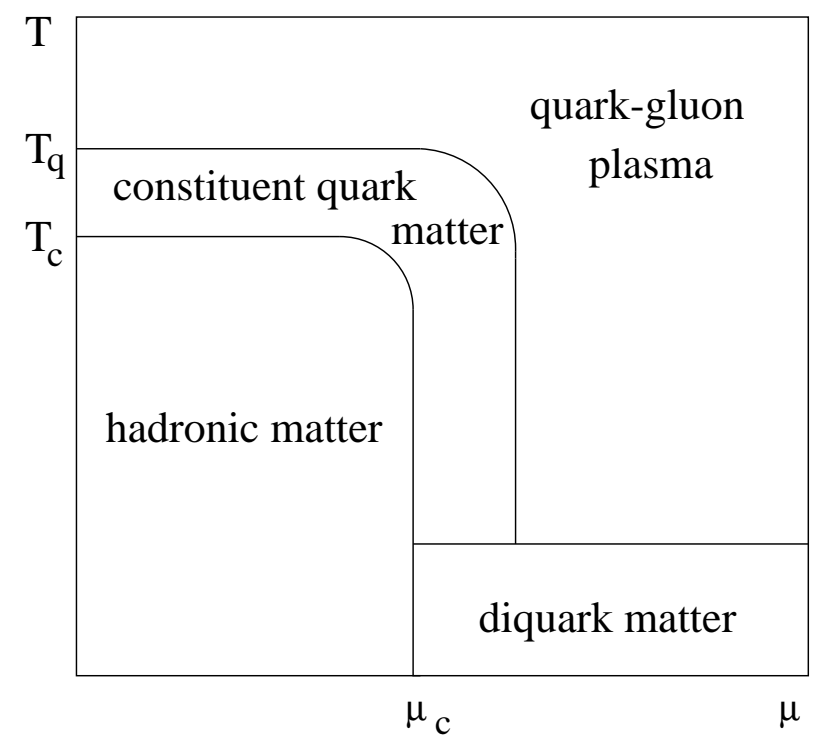

Figure 1: Four-phase diagram of strongly interacting matter.

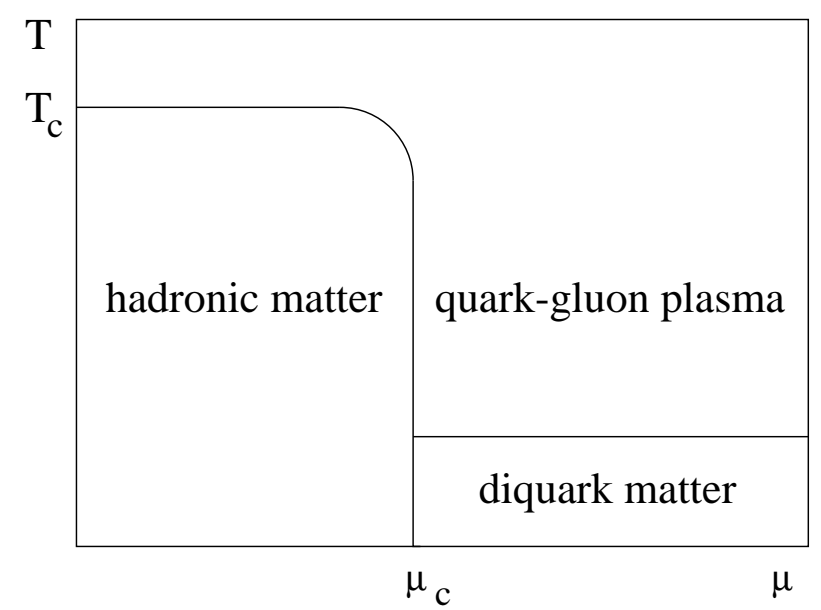

Figure 2: Three-phase diagram of strongly interacting matter. 
The occurrence of such singularities can be attributed to different underlying physics mechanisms. In statistical physics, spontaneous symmetry breaking, charge screening and cluster percolation have received particular attention. In the following sections, I shall consider these three 'mechanisms' in the context of statistical QCD.

\section{Symmetry Breaking}

In contrast to the statistical mechanics of condensed atomic matter, the phase structure of strongly interacting matter can be determined ab initio from QCD as input dynamics, without intermediate models. Given the QCD Lagrange density

$$
\mathcal{L}_{\mathrm{QCD}}=-F_{\mu \nu} F^{\mu \nu}-\bar{\psi}\left(i \gamma^{\mu} \partial_{\mu}-g \gamma^{\mu} A_{\mu}+m_{q}\right) \psi
$$

where the first term describes the pure gluon sector and the second the quark-gluon interactions, one defines the partition function at temperature $T$ as

$$
Z(T, V)=\int d A d \bar{\psi} d \psi \exp \{-S(\mathcal{L}, T)\}
$$

Here $A$ denotes the gluon and $\psi$ the quark fields; the QCD action is given by 10

$$
S(\mathcal{L} ; T, V)=\int_{V} d^{3} x \int_{0}^{1 / T} d \tau \mathcal{L}(A(x, \tau), \psi(x, \tau))
$$

as integral over the volume $V$ of the system and a slice of thickness $1 / T$ in the imaginary time $\tau=i x_{0}$. From $Z(T, V)$ one then obtains the usual thermodynamic functions; the derivative with respect to $T$ leads to the energy density $\epsilon(T)$, that with respect to $V$ the pressure $P(T)$, and so on. Both the dynamic input theory and its associated thermodynamics are thus completely specified; the problem lies in the evaluation, for which one has to resort to the numerical simulation [11] of the lattice formulation [12].

The conventional deconfinement probe in finite temperature lattice QCD is the expectation value $L(T)$ of the Polyakov loop [13, 14]. Through

$$
L(T) \sim \lim _{r \rightarrow \infty} e^{-V(r) / T}
$$

it is related to the potential $V(r)$ coupling a static quark-antiquark pair. In the confined phase, this potential diverges as $V(r)$ for $r \rightarrow \infty$ (see Eq. (3)), while in the deconfined phase it converges to a finite value. It is found that $L(T)=0$ in the temperature range $T \leq T_{c}$, defining the confinement region, and that $L(T)>0$ for $T>T_{c}$, specifying the deconfinement region.

Strictly speaking, $V(r) \sim \sigma r$ diverges for $r \rightarrow \infty$ only in a theory with infinitely heavy quarks. In real QCD with light dynamical quarks, the string breaks when it becomes energetically more favourable to produce a quark-antiquark pair, i.e., when $V(r) \simeq 2 m_{Q}$, where $m_{Q}$ is the mass of a 'dressed' constituent quark and $2 m_{Q}$ the mass of a (nonGoldstone) meson. The quark of this newly produced pair combines with the original antiquark, the antiquark with the original quark, thus making two strings out of one. In 
full QCD, the Polykov loop therefore does not vanish in the confining region, but only becomes exponentially small,

$$
L(T) \simeq e^{-2 m_{Q} / T} \quad \text { for } T \leq T_{c}
$$

For $m_{Q} \simeq 0.3 \mathrm{GeV}$ and $T_{c} \simeq 0.15 \mathrm{GeV}$, this makes $L\left(T_{c}\right) \simeq 0.02$ instead of zero. Nevertheless, $L(T)$ is now no longer a real order parameter, and deconfinement therefore seems to be not really defined as a critical phenomenon in QCD with light dynamical quarks. We shall return to this problem several times and show how it might be solved.

From statistical mechanics, it is known that phase transitions are generally associated to symmetries of the system. Thus the Hamiltonian of the simplest spin theory, the Ising model, is

$$
\mathcal{H}=-J \sum_{i<j} s_{i} s_{j}-B \sum_{i} s_{i}, \quad s_{i}= \pm 1 \forall i,
$$

where the first sum runs only over nearest neighours on the lattice, $J$ denotes the exchange energy between spins and $B$ an external magnetic field. For $B=0, \mathcal{H}$ is invariant under the global $Z_{2}$ symmetry of flipping all spins, $s_{i} \rightarrow-s_{i} \forall i$. The thermodynamic states of this system share this symmetry for $T \geq T_{c}$, where $T_{c}$ now is the Curie point; as a result, the expectation value of the spin, the magnetization $m(T)$, vanishes in this 'disordered' temperature region. Below $T_{c}$, however, the system becomes ordered, the spins choose to align either up or down, making $m(T) \neq 0$. Since 'up' or 'down' are equally likely, the symmetry as such is preserved; the actual state of the system, however, spontaneously breaks it by choosing one or the other. The magnetization transition of the Ising model thus corresponds to the spontaneous breaking of the inherent global $Z_{2}$ symmetry of the Ising Hamiltonian.

This line of argument applies directly also to deconfinement in pure $S U(N)$ gauge theories [13, 14]. The corresponding Lagrangian in lattice QCD

$$
\mathcal{L}_{\mathrm{SU}(\mathrm{N})}\left(U_{i k} U_{k l} U_{l m}^{+} U_{m i}^{+}\right)
$$

depends on the products of four $S U(N)$ matrices $U_{i j}$ on the links of the smallest closed loops of the lattice. It remains invariant under a global 'flip' $z_{N} \in Z_{N} \subset S U(N)$ of all matrices associated to a spatial hyperplane, with $U_{x, \tau} \rightarrow z_{N} U_{x, \tau} \forall x$; here $z_{N}=$ $\exp \{r(2 \pi i / N)\}$ with $r=1,2, \ldots, N$.

The Polyakov loop, on the other hand, does not remain invariant under such global $Z_{N}$ transformations, with

$$
L \sim<\operatorname{Re} \operatorname{Tr} \Pi_{\tau=1}^{N_{\tau}} U_{x, \tau}>\rightarrow z_{N} L
$$

It is thus the analogue of the magnetization of the Ising model, in the sense that it tests if the state of the system shares or spontaneously breaks the symmetry of the Lagrangian. This feature becomes particularly transparent for $S U(2)$ gauge theory, where $z_{2}= \pm 1$, so that the transformation thus just means flipping the sign of the Polyakov loop, $L \rightarrow$ $-L$. In the temperature region in which $L(T)=0$, i.e., in the confinement region, the 
states are $Z_{N}$-symmetric, while for deconfinement, with $L(T)>0$, the $Z_{N}$ symmetry is spontaneously broken. In other words, deconfinement in pure $S U(N)$ gauge theory can be defined as the spontaneous breaking of a global $Z_{N}$ symmetry of the corresponding Lagrangian.

The similarity between spin and gauge systems goes in fact much further [15. In Fig. 3, we compare schematically the temperature behaviour of the Polyakov loop $L(T)$ and the magnetization $m(T)$, together with that of the corresponding susceptibilities $\chi_{L}(T)$ and $\chi_{m}(T)$. The latter measure the fluctuations of the order parameters at the transition point and thus diverge there. For $S U(2)$ gauge theory as well as for the Ising model, the transition is continuous, and so near $T_{c}$ the functional behaviour in the two cases can be written as

$$
L(T) \sim\left(T-T_{c}\right)^{\beta_{L}}, T>T_{c} ; \quad \chi_{L}(T) \sim\left|T-T_{c}\right|^{-\gamma_{L}},
$$

and

$$
m(T) \sim\left(T_{c}-T\right)^{\beta_{m}}, T<T_{c} ; \quad \chi_{m}(T) \sim\left|T-T_{c}\right|^{-\gamma_{m}},
$$

where $\beta$ and $\gamma$ denote the critical exponents for the two transitions. While $S U(N)$ gauge theories in general have a more complex structure than spin theories, their critical behaviour becomes in fact identical: they belong to the same universality class [15], which means that $\beta_{L}=\beta_{m}$ and $\gamma_{L}=\gamma_{m}$. The confinement/deconfinement transition in $S U(N)$ gauge theories is thus structurally the same as the disorder/order transition in spin theories; both are based on the spontaneous breaking of a global $Z_{N}$ symmetry of the underlying dynamics.
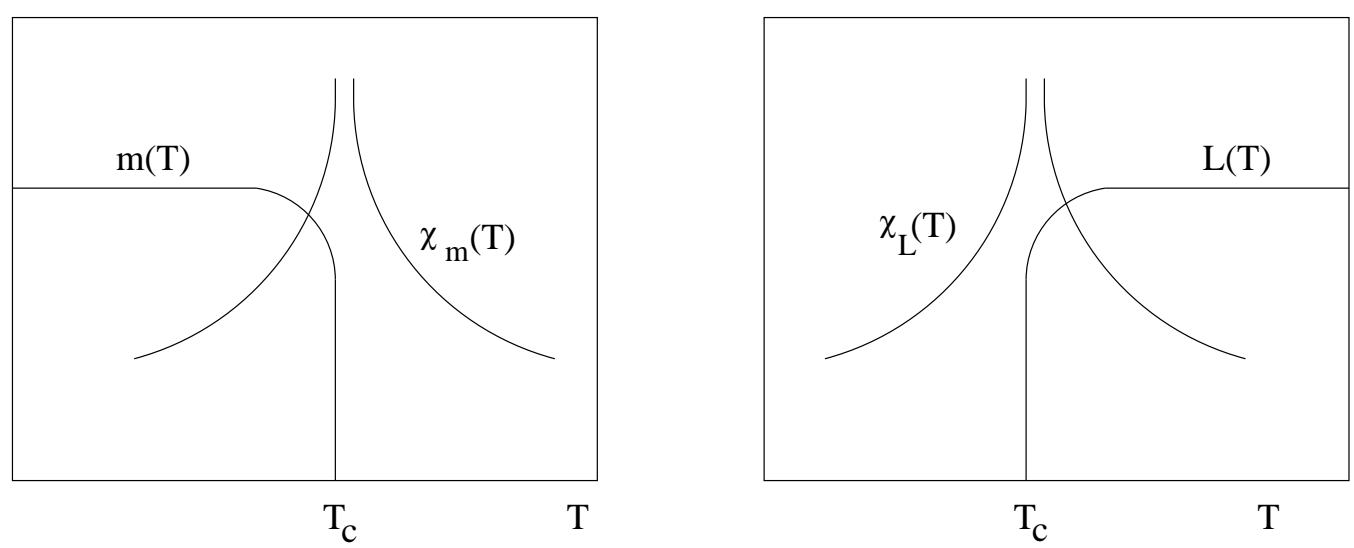

Figure 3: Schematic temperature dependence of the magnetization $m(T)$ and the Polyakov loop $L(T)$, together with the corresponding susceptibilities.

The introduction of dynamical quarks in full QCD explicitly breaks this $Z_{N}$ symmetry; it effectively adds a term to the $S U(N)$ action which is proportional to $L$ :

$$
S_{\mathrm{QCD}} \sim S_{\mathrm{SU}(\mathrm{N})}+\kappa\left(m_{q}\right) L
$$

where $\kappa\left(m_{q}\right) \rightarrow 0$ for $m_{q} \rightarrow \infty$. Comparing Eqs. (16) and (11), we see that dynamical quarks in a sense play the role of an external field $B$ in spin theory. Just as $B$ aligns the 
spins and prevents $m(T)$ from ever completely vanishing, so does $m_{q}$ result in a Polyakov loop which is always non-zero. We had seen above that another way of arriving at this conclusion is through string breaking. Hence there must be some implicit relation between the effective external field and the constituent quark mass determining the string breaking point. The effect of dynamical quarks on the Polyakov loop is shown schematically in Fig. ๒. We note in particular that even for $m_{q}=0$, there is 'almost critical' behaviour, with a sharp variation at a temperature considerably below the deconfinement temperature of pure $S U(3)$ gauge theory What are the reasons for this behaviour?

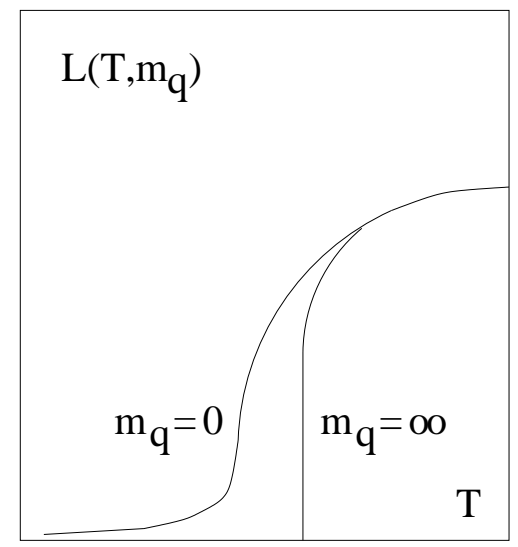

Figure 4: Schematic temperature dependence of the Polyakov loop in pure $S U(3)$ gauge theory $\left(m_{q}=\infty\right)$ and in full QCD with two massless quark flavours $\left(m_{q}=0\right)$.

From the string breaking picture, we expect the effective external field acting on the Polyakov loop as generalized spin to be inversely proportional to the constituent quark mass, $B \sim 1 / m_{Q}$ [16, 17, 18]. Thus deconfinement should occur when $m_{Q} \rightarrow 0$.

For $m_{q}=0$, the QCD Lagrangian is chirally symmetric; however, the state of the system under given conditions need not share this symmetry. The chiral condensate, $K(T) \equiv\langle\psi \bar{\psi}\rangle \sim m_{Q}^{3}$, provides an order parameter to probe if and when the chiral symmetry of the Lagrangian is spontaneously broken. It is found that

$$
K(T) \neq 0 \quad \text { implying } \quad m_{Q} \neq 0 \quad \forall T<T_{\chi},
$$

and

$$
K(T)=0 \quad \text { implying } \quad m_{Q}=0 \quad \forall T>T_{\chi} .
$$

where $T_{\chi} \mathrm{MeV}$ is the chiral symmetry restoration temperature. The functional behaviour of $K$ and the corresponding fluctuation susceptibility $\chi_{K}$,

$$
K(T) \sim\left(T_{\chi}-T\right)^{\beta_{K}}, T<T_{\chi} ; \quad \chi_{K}(T) \sim\left|T-T_{\chi}\right|^{-\gamma_{K}},
$$

is illustrated in Fig. 5. For $T<T_{\chi}, K$ is large and hence the effective external field $B \sim 1 / m_{Q} \sim 1 / K(T)$ is small, so that the Polyakov loop is almost disordered, implying confinement-like behaviour. At $T=T_{\chi}, B$ suddenly becomes large; it aligns the Polyakov 


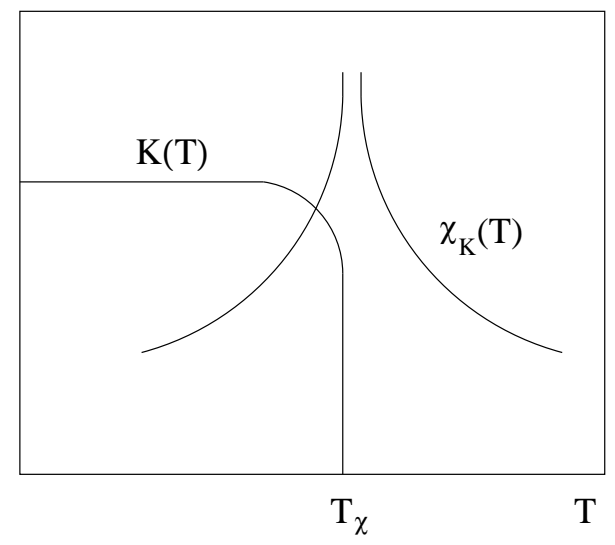

Figure 5: Schematic temperature dependence of the chiral condensate $K(T)$ in full QCD, together with the corresponding susceptibility.

loops, implying the onset of deconfinement. We thus find that chiral symmetry restoration induces colour deconfinement.

These considerations have immediate consequences which can be tested in finite temperature lattice QCD. The magnetization $m(T, B)$ for non-vanishing external field $B$ becomes an analytic function of $T$ and $B$. In full QCD, we therefore assume the Polyakov loop for $m_{q} \neq 0$ to be an analytic function of $T$ and $K$. This leads to

$$
d L=\left(\frac{\partial L}{\partial T}\right)_{K} d T+\left(\frac{\partial L}{\partial K}\right)_{T} d K
$$

From this we obtain

$$
\chi_{m}^{L} \equiv\left(\frac{\partial L}{\partial m_{q}}\right)_{T}=\left(\frac{\partial L}{\partial K}\right)_{T}\left(\frac{\partial K}{\partial m_{q}}\right)_{T},
$$

and

$$
\chi_{T}^{L} \equiv\left(\frac{\partial L}{\partial T}\right)_{m_{q}}=\left(\frac{\partial L}{\partial K}\right)_{T}\left(\frac{\partial K}{\partial T}\right)_{m_{q}}+\left(\frac{\partial L}{\partial T}\right)_{K}
$$

for the Polyakov loop susceptibilities $\chi_{m}^{L}$ and $\chi_{T}^{L}$. Since the chiral susceptibilities $\chi_{m}^{K}=$ $\left(\partial K / \partial m_{q}\right)_{T}$ and $\chi_{T}^{K}=(\partial K / \partial T)_{m_{q}}$ diverge at $T=T_{\chi}$, relations (21/22) imply that the Polyakov loop susceptibilities must share this singular behaviour, with the same critical exponents. Present lattice calculations for full QCD are not yet precise enough to allow a conclusive determination of critical exponents. In Figs. 6 and 7 it is seen, however, that the increase of the chiral susceptibilities $\chi_{m}^{K}$ and $\chi_{T}^{K}$ for $m_{q} \rightarrow 0$ is indeed accompanied by a similar increase in the Polyakov loop susceptibilities $\chi_{m}^{L}$ and $\chi_{T}^{L}$.

In QCD with massless dynamical quarks, the chiral condensate $K$ and the Polyakov loop $L$ thus are analytically related; there is only one critical point $T=T_{\chi}$, at which both $K(T)$ and $L(T)$ exhibit non-analytic behaviour. At $T_{\chi}$, the quarks loose their effective mass and at the same time become unbound. 

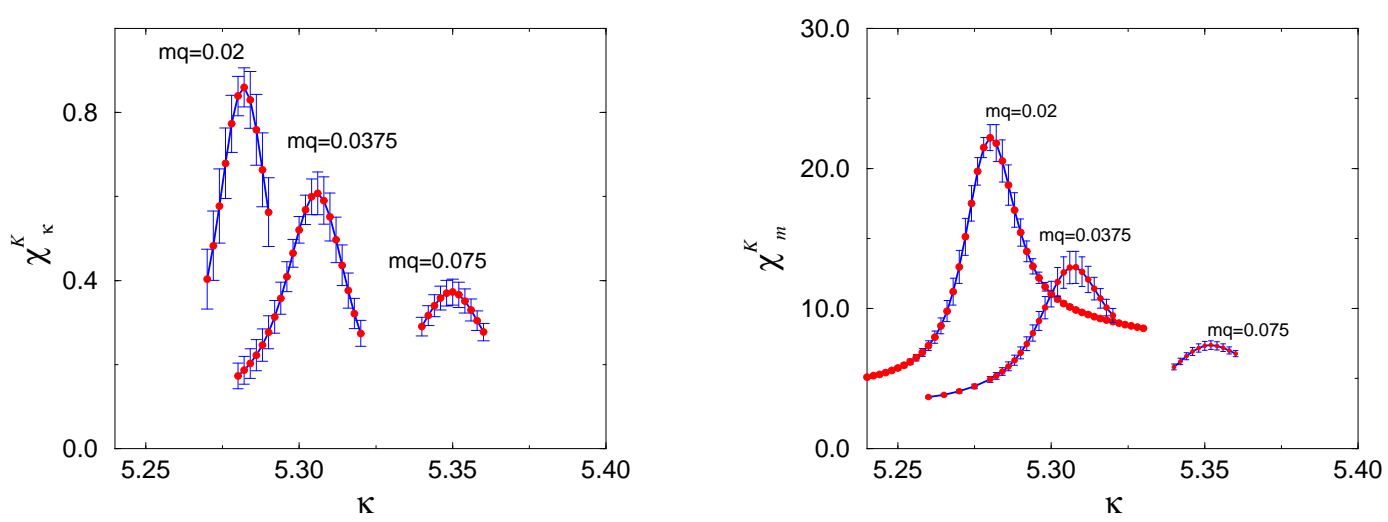

Figure 6: The chiral susceptibilities $\chi_{\kappa}^{K}$ and $\chi_{\kappa}^{K}$ as functions of the temperature variable $\kappa=6 / g^{2}$.
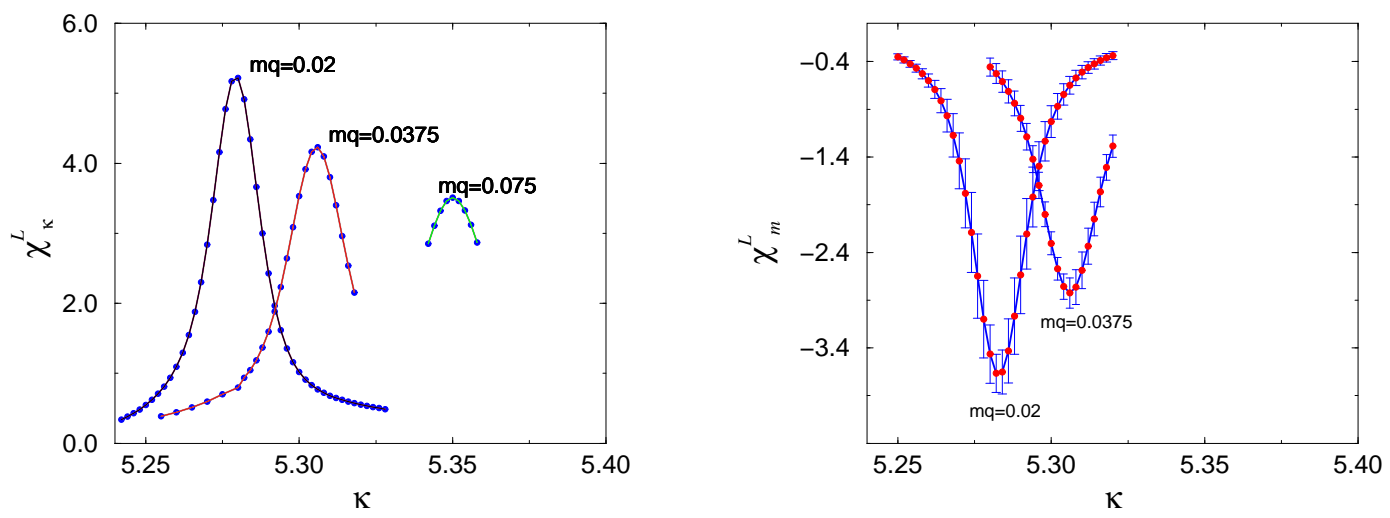

Figure 7: The Polyakov loop susceptibilities $\chi_{\kappa}^{L}$ and $\chi_{\kappa}^{L}$ as functions of the temperature variable $\kappa=6 / g^{2}$.

\section{Colour Screening}

In pure $S U(N)$ gauge theory, the potential between a static quark and antiquark increases linearly and unbounded with their separation $r, V(r) \sim \sigma r$. In full QCD, the string breaks when $V(r) \simeq m_{Q}$, even in vacuum, i.e., at $T=0$. We can interpret this by attributing a screening behaviour to the sea of virtual massless quark-antiquark pairs. Starting from Eq. (11), we thus have

$$
V(r, \mu)=\sigma r\left[\frac{1-e^{-\mu r}}{\mu r}\right]-\frac{\alpha}{r} e^{-\mu r},
$$


where the last term includes Coulomb and transverse string effects. In Eq. (23), the string tension $\sigma$ and the Coulomb coupling $\alpha$ are taken to be constants, with $\mu(T)$ temperaturedependent. First, we now want to determine the vacuum screening mass $\mu(T=0)$.

In the spectroscopy of heavy quarkonia, such as the $J / \psi$ or the $\Upsilon$, the masses and widths of all bound states are determined by solving the Schrödinger equation

$$
\mathcal{H} \phi_{i}=M_{i} \phi_{i}
$$

with the Hamiltonian

$$
\mathcal{H} \equiv 2 m_{c, b}-\frac{\nabla^{2}}{m_{c, b}}+V(r, \mu=0)
$$

given in terms of the potential (23). Here $i$ specifies the $c \bar{c}$ or $b \bar{b}$ bound state under consideration, $M_{i}$ its mass. By comparison for the results to quarkonium data, the four constants in the potential are determined, giving $\sigma=0.192 \mathrm{GeV}^{2}, \alpha=0.471$ and $m_{c}=$ $1.32 \mathrm{GeV}, m_{b}=4.75 \mathrm{GeV}$ for the bare charm and bottom quark masses, respectively. The vacuum screening mass can now be obtained by comparing the gap between the open charm or bottom threshold, $2 M_{D}$ or $2 M_{B}$, and a given quarkonium state $M_{i}$, to its gap with respect to the infinite range potential,

$$
E_{\mathrm{diss}}^{i}=2 M_{D, B}-M_{i}=2 m_{c, b}+\frac{\sigma}{\mu}-M_{i} .
$$

The result,

$$
\mu=\frac{\sigma}{2\left(M_{D, B}-m_{c, b}\right)}
$$

gives $\mu(T=0) \simeq 0.18 \mathrm{GeV}$ for both charmonium and bottonium states. The fact that the large difference between $m_{c}$ and $m_{b}$ plays no role here is an indication that the states are indeed heavy enough to estimate the medium effect alone. It is moreover reassuring that the 'Debye' screening length $r_{D}=\mu(T=0) \simeq 1.1 \mathrm{fm}$ is also the expected hadronic scale.

At non-vanishing temperature, the screening mass can be determined through a study of Polyakov loop correlations $\left\langle L(0) L^{+}(r)\right\rangle$. Normalization problems make this non-trivial [19], so that for the moment $\mu(T)$ is known only up to an open constant. It is already clear, however, that $\mu(T)$ increases sharply around $T=T_{\chi}$, with $(\partial \mu / \partial T)$ diverging in the chiral limit. Combining this result with the known $\mu(T=0)$ and the perturbative result $\mu \sim g T$ leads to the schematic screening mass form shown in Fig. 8. In QCD, the screening mass thus shows a very characteristic behaviour, with a singular derivative at $T_{\chi}$.

The physics leading to this phenomenon seems quite clear. Chiral symmetry restoration transforms effectively massive into massless quarks. Near $T=T_{\chi}$, this sudden loss of mass leads to a sudden increase in the density $n$ of coloured constituents and thus, with $\mu \sim n^{1 / 3}$, also in the effectiveness of colour screening.

An interesting side-line here is the onset of charmonium suppression as signal for colour deconfinement [20]. Deconfined media will dissociate charmonium and bottonium 


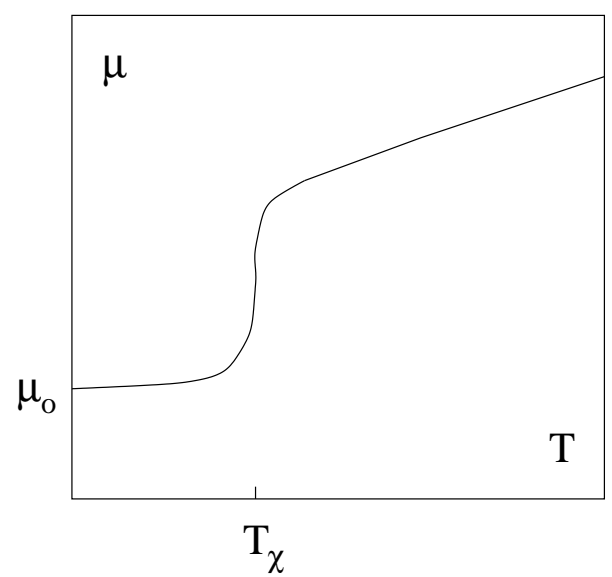

Figure 8: Schematic temperature dependence of the screening mass $\mu$ in full QCD with massless quarks of two flavours.

states in a step-wise pattern, with the larger and more loosely bound states melting before smaller and more tightly bound quarkonia. There are indications that the dissociation point for the charmonium states $\chi_{c}(1 P)$ and $\psi^{\prime}(2 S)$ coincides with $T_{\chi}$ [21]. If this could be substantiated in more precise lattice studies, it would identify the onset of $\chi_{c}$ and $\psi^{\prime}$ suppression with the onset of colour deconfinement.

\section{Cluster Percolation}

Conceptually, the deconfinement transition seems rather transparent, no matter what the quark mass is. Once the density of constituents becomes so high that several hadrons have considerable overlap, there is no mechanism to partition the quark constituents into colour-neutral bound states. Instead, there appear clusters much larger than hadrons, within which colour is not confined. This suggests that deconfinement is related to cluster formation, and since that is the central topic of percolation theory, possible connections between percolation and deconfinement were discussed already quite some time ago on a rather qualitative level [22, 23]. In the meantime, however, the interrelation of geometric cluster percolation and critical behaviour of thermal systems has become much better understood [24], and this understanding can be used to clarify the nature of deconfinement.

To recall the fundamentals of percolation, consider a two-dimensional square lattice of linear size $L$; we randomly place identical objects on $N$ of the $L^{2}$ lattice sites. With increasing $N$, adjacent occupied sites will begin to form growing clusters or islands in the sea of empty sites. Define $n_{p}$ to be the lowest value of the density $n=N / L^{2}$ for which on the average the origin belongs to a cluster reaching the edge of the lattice. In the limit $L \rightarrow \infty$, we then have

$$
P(n) \sim\left(1-\frac{n_{p}}{n}\right)^{\beta_{p}}, \quad n \geq n_{p}
$$

where the percolation strength $P(n)$ denotes the probability that the origin belongs to an infinite cluster. Since $P(n)=0$ for all $n \leq n_{p}$ and non-zero for all $n>n_{p}$, it constitutes 
an order parameter for percolation: $\beta_{p}=5 / 36$ is the critical exponent which governs the vanishing of $P(n)$ at $n=n_{p}$ in two dimensions; in three dimensions, it becomes $\beta=0.41$ 25.

Another quantity of particular interest is the average cluster size $S(n)$, defined as the average number of connected occupied sites containing the origin of the lattice; above $n_{p}$, percolating clusters are excluded in the averaging. This cluster size corresponds to the susceptibility in thermal systems and diverges at the percolation point as

$$
\sigma_{c \bar{c}}(n) \sim\left|n-n_{p}\right|^{-\gamma_{p}}
$$

with $\gamma_{p}=43 / 18(1.80)$ as the $d=2(3)$ critical exponent for the divergence [25].

We now turn once more to the Ising model. For $B=0$, the Hamiltonian (11)) has a global $Z_{2}$ invariance $\left(s_{i} \rightarrow-s_{i} \forall i\right)$, and the magnetization $m=\langle s\rangle$ probes whether this invariance is spontaneously broken, as discussed in section 2. Such spontaneous symmetry breaking occurs below the Curie point $T_{c}$, with

$$
m(T, B=0) \sim\left(T_{c}-T\right)^{\beta_{m}}
$$

governing the vanishing of $m(T, B=0)$ as $T \rightarrow T_{c}$ from below. The well-known Onsager calculations with $\beta_{m}=0.125$ for $d=2$, a value $10 \%$ smaller than the $\beta_{p}=5 / 36 \simeq 0.139$ found for the percolation exponent.

Since the Ising model also produces clusters on the lattice, consisting of connected regions of aligned up or down spins, the relation between its thermal critical behaviour at $T_{c}$ and the onset of geometric percolation is an obvious question which has been studied extensively in recent years. In other words, can one interpret magnetization as spin domain fusion? This question is now answered [26, 27].

The geometric clusters in a percolation study consist of connected regions of spins pointing in the same direction. In the Ising model, there is a thermal correlation between spins on different sites; this vanishes for $T \rightarrow \infty$. Correlated regions in the Ising model (we follow the usual notation and call them 'droplets', to distinguish them from geometric clusters) thus disappear in the high temperature limit. In contrast, the geometric clusters never vanish, since the probability for a finite number of adjacent aligned spins always remains finite; it increases with dimension because the number of neighbours does. Hence from the point of view of percolation, there are more and bigger clusters than there are Ising droplets.

If percolation is to provide the given thermal critical behaviour, the definition of cluster has to be changed such that the modified percolation clusters coincide with the correlated Ising droplets [26, 27]. This is achieved by assigning to pairs of adjacent aligned spins in a geometric cluster an additional bond correlation, present with the bonding probability

$$
p_{b}=1-\exp \{-2 J / k T\}
$$

where $2 J$ corresponds to the energy required for flipping an aligned into a non-aligned spin. The modified 'F-K' percolation clusters now consist of aligned spins which are also 
bond-connected. Only for $T=0$ are all aligned spins bonded; for $T>0$, some aligned spins in a purely geometric cluster are not bonded and hence do not belong to the modified cluster or droplet. This effectively reduces the size of a given geometric cluster or even cuts it into several modified clusters. For $T \rightarrow \infty, n_{b} \rightarrow 0$, so that the geometric clusters still in existence there are not counted as droplets, solving the problem mentioned in the previous paragraph.

For such combined F-K site/bond clusters, full agreement between percolation and thermal critical behaviour of the Ising model is achieved for any space dimension $d$. The percolation threshold is now at $T_{c}$, the cluster size coincides with that of the correlated regions in the Ising model, and numerical simulations show that the critical exponents for the new cluster percolation scheme become those of the Ising model.

Since, as noted, the deconfinement transition in $S U(N)$ gauge theory falls into the universality class of the Ising model, it seems natural to look for a percolation formulation of deconfinement [17], and first studies indicate that this is indeed possible [31, 32]. In $S U(2)$ lattice gauge theory, the Polyakov loop constitutes essentially a generalized spin variable, pointing either up or down at each spatial lattice site, but with continuously varying magnitude. In two space dimensions, this leads to a 'landscape' of hills and lakes of various heights and depths. The crucial question in the extension of percolation to such a case is how to generalize the bond weight Eq. (31). For a specific lattice regularization, the strong coupling limit, it was shown that the action in $S U(2)$ gauge theory can effectively be written in terms of nearest neighbour Polyakov loop interactions, with $(\kappa / 4)^{2} L_{i} L_{j}$ in place of the Ising form $(J / k T) s_{i} s_{j}$, where $\kappa \equiv 4 / g^{2}$ and $g$ denotes the coupling in the gauge theory action [34]. We therefore take

$$
p_{i, j}^{b}=1-\exp \left\{-2(\kappa / 4)^{2} L_{i} L_{j}\right\}
$$

as bond weight between two adjacent Polyakov loops of the same sign. It is known through analytic as well as numerical studies that such a form gives the correct bond weight for continuous spin Ising models [35, 36]. To test it here, we have carried out studies on a number of different lattices for both two and three space dimensions; some results are shown in Fig. 9 and in Table 1. Fig. 9 shows that the rescaled percolation probability, using the Ising value for the exponent $\nu$ leads to a universal curve, as required. In the table we summarize the excellent agreement between thermal and percolation values for the critical exponents. The exponents for random site percolation, on the other hand, are seen to be considerably different.

For the specific lattice regularization used, we thus indeed find that deconfinement in $S U(2)$ gauge theory can be described as Polyakov loop percolation. Hopefully this can be extended to more general lattice regularizations.

The greatest interest in a percolation approach to deconfinement is, however, based on the possibility to define the transition for arbitrary values of the quark mass. Consider the case of full QCD with colour $S U(3)$ and two massless quark flavours. For $m_{q} \rightarrow \infty$, this leads to the first order transition of $S U(3)$ gauge theory, as counterpart of such a transition in the three-state Potts model. For decreasing quark mass, the transition will eventually 


\begin{tabular}{llll}
\hline & \multicolumn{1}{c}{$\beta / \nu$} & \multicolumn{1}{c}{$\gamma / \nu$} & \multicolumn{1}{c}{$\nu$} \\
\hline L-Percolation & $0.528(15)$ & $1.985(13)$ & $0.632(11)$ \\
Symmetry Breaking & $0.523(12)$ & $1.953(18)$ & $0.630(14)$ \\
Ising Model [33] & $0.518(7)$ & $1.970(11)$ & $0.6289(8)$ \\
Random Percolation [37] & $0.4770(10)$ & $2.0460(39)$ & $0.8765(16)$ \\
\hline
\end{tabular}

Table 1: Comparison of percolation and thermal exponents for $3+1 S U(2)$.

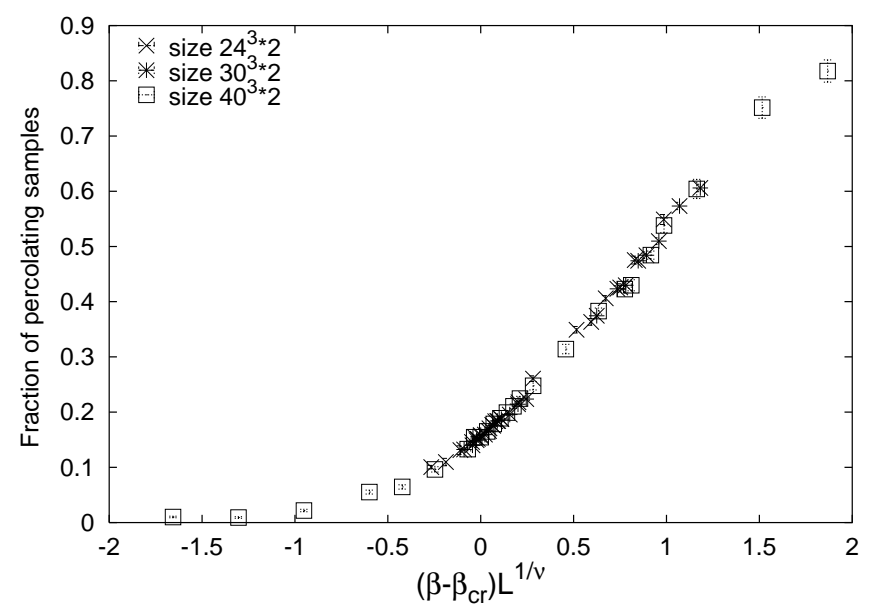

Figure 9: Rescaled percolation probability for $3+1 S U(3)$ gauge theory, using the Ising exponent $\nu=1$.

disappear at a second-order end point defined in terms of $m_{q}^{c}$, and for $m_{q}^{c}>m_{q}>0$, there presumably is no thermal transition at all. Finally, for $m_{q} \rightarrow 0$, there is the chiral symmetry restoration transition. In Fig. 10, this behaviour is illustrated. Does this mean that in the quark mass region $m_{q}^{c}>m_{q}>0$ (which includes our real physical world of small but finite bare quark masses) there is no way to define deconfinement as a critical phenomenon?

To address this question, we return to percolation in the Ising model with a nonvanishing external field. For $H \neq 0$, the Ising partition function does not contain any singularity as function of $T$ and hence does not show any critical behaviour [30]; the $Z_{2}$ symmetry responsible for the onset of spontaneous magnetization is now always broken and $m(T, H \neq 0) \neq 0$ for all $T$. On the other hand, the average size of site/bond clusters in the above sense increases with decreasing temperature, and above some critical temperature it diverges. Hence percolation will occur for any value of $B$. In other words, the critical behaviour due to percolation persists, while that related to spontaneous symmetry breaking and magnetization disappears. At $B=\infty$, all spins are aligned, leaving the bonds as the relevant variables; the system now percolates at the critical density for pure 


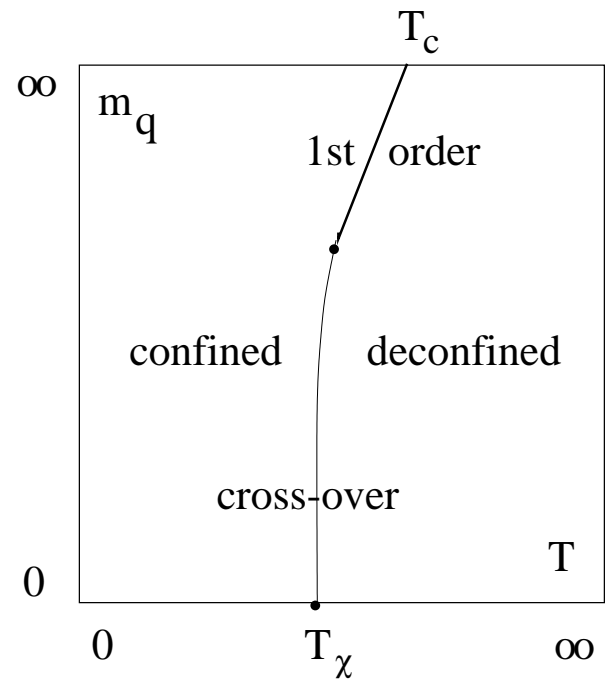

Figure 10: QCD phase structure as function of temperature $T$ and quark mass $m_{q}$.

bond percolation, which leads to a critical temperature $T_{k}$ somewhat above the Curie point $T_{c}$. For finite $B$, the corresponding values of the critical temperature lie between $T_{c}$ at $B=0$ and $T_{k}$ at $B=\infty$; they define the so-called Kertész line 24, 28]; see Fig. 11. A fundamental and quite general question in statistical physics is what happens at this line. Can one generalize critical behaviour to situations where the partition function $Z(T)$ is analytic, but where percolation as defined in terms of the input dynamics persists? It is evident from the similarity of Figs. 10 and 11 that the answer is immediately relevant to the study of phase transitions in QCD, with deconfinement as the QCD counterpart of the Kertész line.

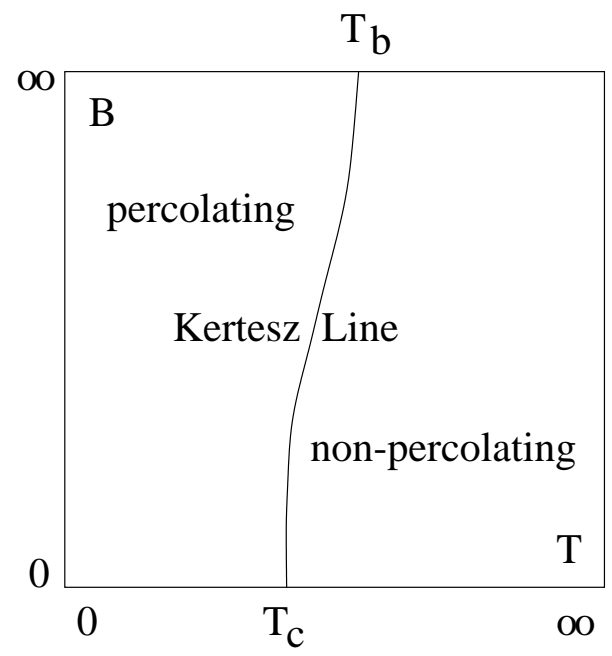

Figure 11: Percolation pattern for the Ising model with external magnetic field $B$.

In statistical physics, such generalized critical behaviour indeed leads to observable effects. Familiar instances are found in solution/gel transitions, as encountered in the 
boiling of an egg or the making of pudding. While these phenomena do not result in any thermodynamic singularities, they are well-defined transitions which can be quantitatively described in terms of percolation [38.

In closing, we note another possible illustration of the relation between percolation and thermal critical behaviour. Consider the F-K site/bond percolation in the two-dimensional Ising model as introduced above, and imagine that current can flow between two or more bonded sites. In this case, conductivity sets in at the percolation point, independent of the magnetization critical behaviour of the Ising model; the system is non-conducting below the percolation point and conducting above it. In other words, we now have two independent critical phenomena, the onset of conductivity and the critical behaviour of Ising thermodynamics, and the former can survive even when the latter is no longer present.

\section{Summary}

We have seen that hadronic matter at sufficiently high temperature and low baryon density becomes a quark-gluon plasma. In this deconfinement transition, the colourneutral bound-state constituents are dissolved into their coloured components. At high baryon density and low temperature, the deconfined medium could be a condensate of coloured bosonic diquarks.

In pure $S U(N)$ gauge theory, colour deconfinement arises through the spontaneous breaking of a global $Z_{N}$ symmetry of the Lagrangian. In the chiral limit of full QCD, it occurs through a strong explicit breaking of this symmetry, due to an effective external field setting in when the chiral condensate vanishes. Hence the deconfinement and chiral symmetry restoration transitions coincide.

Colour charge screening in QCD leads to a specific singular behaviour of the screening mass. At the critical temperature of chiral symmetry restoration, the effective quark mass shift leads to sudden increase in the density of constituents and hence to more effective screening. A particularly enticing question here is whether the dissociation of the $\chi_{c}$ state occurs at just this point - it would then be a measurable order parameter for deconfinement.

Cluster percolation provides an approach to study the geometry of deconfinement. In $S U(N)$ gauge theory, first finite temperature lattice calculations indicate that Polyakov loop percolation is indeed an equivalent way to identify deconfinement. In full QCD as in spin systems with non-vanishing external field, percolation persists even in the absence of thermal transitions. It thus seems conceivable to identify the colour deconfinement transition for arbitrary quark mass through the onset of percolation.

\section{Acknowledgements}

It is a pleasure to thank Ph. Blanchard, S. Digal, S. Fortunato, D. Gandolfo, F. Karsch, E. Laermann and P. Petreczky for helpful discussions on different aspects of this survey. 


\section{References}

[1] I. Ya. Pomeranchuk, Doklady Akad. Nauk. SSSR 78 (1951) 889.

[2] L. Euler, Novi Commentarii Academiae Scientiarum Petropolitanae 3 (1753) 125;

E. Schröder, Z. Math. Phys. 15 (1870) 361;

G. H. Hardy and S. Ramanujan, Proc. London Math. Soc. 17 (1918) 75.

[3] R. Hagedorn, Nuovo Cim. Suppl. 3 (1965) 147.

[4] S. Fubini and G. Veneziano, Nuovo Cim. 64 A (1969) 811;

K. Bardakçi and S. Mandelstam, Phys. Rev. 184 (1969) 1640.

[5] N. Cabibbo and G. Parisi, Phys. Lett. 59B (1975) 67.

[6] N. F. Mott, Proc. Phys. Soc. (London) A62 (1949) 416.

[7] V. V. Dixit, Mod. Phys. Lett. A 5 (1990) 227.

[8] D. Bailin and A. Love, Phys. Rept. 107 (1984) 325, and further references there.

[9] For a recent survey, see K. Rajagopal, Nucl. Phys. A 661 (1999) 150c.

[10] C. Bernard, Phys. Rev. D 9 (1974) 3312.

[11] M. Creutz, Phys. Rev. D 21 (1980) 2308.

[12] K. G. Wilson, Phys. Rev. D 10 (1974) 2445.

[13] L. D. McLerran and B. Svetitsky, Phys. Lett. 98 B (1981) 195 and Phys. Rev. D 24 (1981) 450 .

[14] J. Kuti, J. Polónyi and K. Szlachányi, Phys. Lett. 98B (1981) 199.

[15] B. Svetitsky and L. G. Yaffe, Nucl. Phys. B 210 [FS6] (1982) 423.

[16] R. V. Gavai, A. Gocksch, and M. Ogilvie, Phys. Rev. Lett. 56 (1986) 815.

[17] H. Satz, Nucl. Phys. A642 (1998) 130c.

[18] S. Digal, E. Laermann and H. Satz, hep-ph/0007175.

[19] S. Digal, E. Laermann and H. Satz, in progress.

[20] T. Matsui and H. Satz, Phys. Lett. 178B (1986) 416.

[21] F. Karsch, M. T. Mehr and H. Satz, Z. Phys. C 37 (1988) 617.

[22] G. Baym, Physica 96A (1979) 131.

[23] T. Çelik, F. Karsch and H. Satz, Phys. Lett. 97B (1980) 128. 
[24] For a recent survey, see D. Stauffer and A. Aharony, Introduction to Percolation Theory, Taylor \& Francis, London 1994.

[25] For surveys, see e. g.

M. B. Isichenko, Rev. Mod. Phys. 64 (1992) 961;

C. D. Lorenz and R. M. Ziff, Phys. Rev. E 57 (1998) 230.

[26] C. M. Fortuin and P. W. Kasteleyn, Physica 57 (1972) 536.

[27] A. Coniglio and W. Klein, J. Phys. A 13 (1980) 2775.

[28] J. Kertész, Physica A 161 (1989) 58.

[29] R. H. Swendsen and J.-S. Wang, Phys. Rev. Lett. 58 (1987) 86.

[30] T. D. Lee and C. N. Yang, Phys. Rev. 87 (1952) 404.

[31] S. Fortunato and H. Satz, Phys. Lett. B475 (2000) 311.

[32] S. Fortunato and H. Satz, hep-lat/0007012, to appear in Nucl. Phys. A.

[33] A. M. Ferrenberg and D. P. Landau, Phys. Rev. B 44 (1991) 5081.

[34] F. Green and F. Karsch, Nucl. Phys. B 238 (1984) 297.

[35] P. Bialas et al., hep-lat/9911020, to appear in Nucl. Phys. B.

[36] S. Fortunato and H. Satz, hep-lat/0007005 (July 2000).

[37] H. G. Ballesteros et al., J. Phys. A 32 (1999) 1.

[38] A. Coniglio, private communication. 